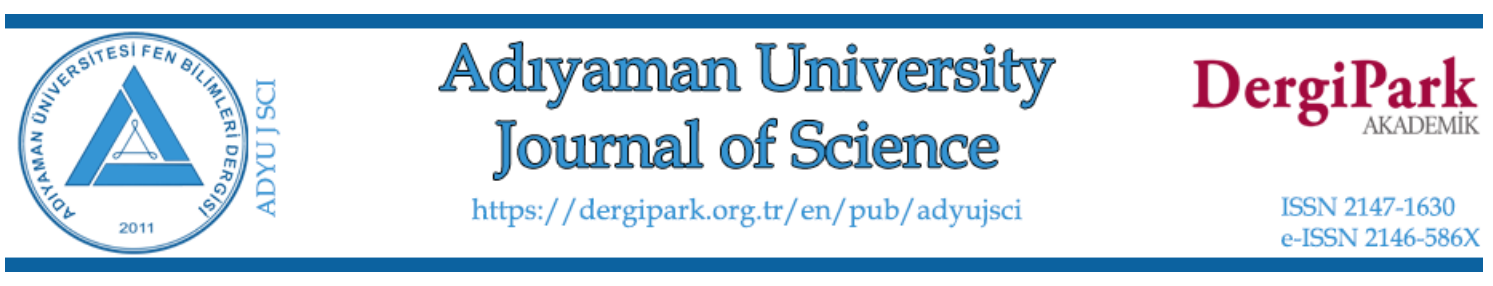

\title{
Increasing the Efficiency of Percentile Parameter Estimation Method for Weibull
}

\section{Distribution}

\author{
Ülkü ERIŞOĞLU1 ${ }^{1}$, Murat ERIŞSOĞLU², Tayfun SERVi̇ ${ }^{3, * *}$ \\ ${ }^{I}$ Necmettin Erbakan University, Faculty of Science, Department of Statistics, Konya, Turkey \\ ugokal@erbakan.edu.tr, ORCID:0000-0002-9826-3460 \\ ${ }^{2}$ Necmettin Erbakan University, Faculty of Science, Department of Statistics, Konya, Turkey \\ merisoglu@erbakan.edu.tr,ORCID:0000-0002-4589-1383 \\ ${ }^{3}$ Adiyaman University, Faculty of Economics and Administrative Science of Science, Department of \\ Economics, Adlyaman, Turkey \\ tservi@adiyaman.edu.tr, ORCID: 0000-0002-3173-327X
}

\begin{abstract}
The Weibull distribution is one of the most widely used probability distributions in statistical applications. The percentile parameter estimation method is commonly used in parameter estimation of the two-parameter Weibull distribution in terms of easy computability and efficiency. The effectiveness of the percentile method depends on the selected percentile points and chosen empirical distribution function. In this study, the appropriate empirical distribution function and percentile points were determined to increase the efficiency of the percentile method for two-parameter Weibull distribution by simulation study.
\end{abstract}

Keywords: Weibull distribution; Percentile estimation; Empirical distribution function.

Weibull Dağılımı için Persentil Parametre Tahmin Yönteminin Etkinliğinin Artırılması

$\ddot{O} \mathbf{z}$ 
Weibull dağılımı istatistiksel uygulamalarda en yaygın kullanılan olasılık dağılımlarından biridir. Persentil parametre tahmin yöntemi kolay hesaplanama ve etkinlik açısından iki parametreli Weibull dağılımının parametre tahmininde yaygın olarak kullanılır. Persentil yönteminin etkinliği seçilen persentil noktalara ve seçilen ampirik dağılım fonksiyonuna bağlıdır. $\mathrm{Bu}$ çalışmada, simülasyon çalışması ile iki parametreli Weibull dağılımı için persentil yönteminin etkinliğini artırmak amacıyla uygun ampirik dağılım fonksiyonu ve persentil noktaları belirlenmiştir.

Anahtar Kelimeler: Weibull dağılımı; Persentil tahmin; Ampirik dağılım fonksiyonu.

\section{Introduction}

The Weibull distribution is one of the widely used probability distributions which has many different applications and used for solving a variety of problems from many different disciplines. The Weibull distribution has many useful properties because its form is flexible to model different shapes. Weibull distribution has increasing, decreasing or stable failure rates depending on the values of the shape parameter. This feature has provided widespread use of the Weibull distribution. Because of its importance, many different estimation methods have been proposed for estimating parameters of Weibull distribution.

Percentile method is one of the commonly used methods for estimating the parameters of Weibull distribution and has some advantages unlike the other estimation methods in terms of ease compute and efficiency in parameter estimation [1].

The percentile method may be applied via two different approaches. The approach 1 is based on percentiles and is structurally similar to the traditional method of moments. The approach 2 is mainly obtained by minimizing the squared Euclidean distance between the sample percentile and population percentile [2].

The effectiveness of the percentile estimators depends on the selected percentile points and chosen empirical distribution function.

Dubey [3] proposed 17th and 97th percentiles for shape parameter and 40th and 82th percentiles for scale parameter. Seki and Yokoyama [4] suggested 31st and 63rd percentiles to estimate shape and scale parameters. Wang and Keats [5] proposed 15th and 63rd percentiles for parameter estimation of the Weibull distributions. Marks [6] stated that the best results were obtained with 10th and 90th percentiles for the shape and scale parameters. 
In this study, we aimed to determine the appropriate empirical distribution and the percentile points to increase the effectiveness of the percentile estimators for the Weibull distribution. We used mean squared error (MSE) and mean total error (MTE) as performance criteria in the simulation study.

\section{Weibull Distribution}

The two-parameter Weibull distribution is specified by the probability density function (pdf)

$$
f(x ; \alpha, \beta)=\frac{\alpha}{\beta}\left(\frac{x}{\beta}\right)^{\alpha-1} e^{-\left(\frac{x}{\beta}\right)^{\alpha}}
$$

where $\alpha>0$ and $\beta>0$ are the shape and scale parameters, respectively. The corresponding cumulative distribution function (cdf) and survival function (sf) are given as bellow:

$$
\begin{aligned}
& F(x ; \alpha, \beta)=1-e^{-\left(\frac{x}{\beta}\right)^{\alpha}}, \\
& S(x ; \alpha, \beta)=e^{-\left(\frac{x}{\beta}\right)^{\alpha}} .
\end{aligned}
$$
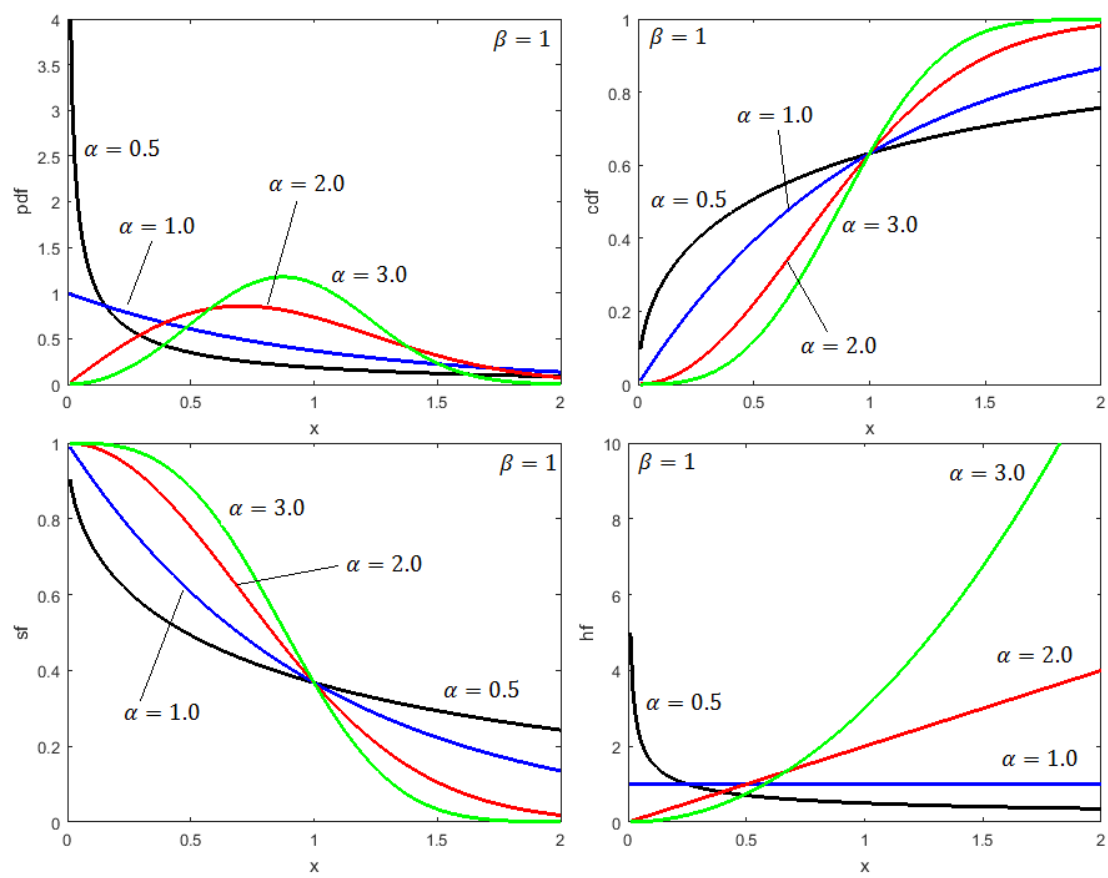

Figure 1: The curves of the pdf, cdf, sf and hf of Weibull distribution for selected parameter values

The hazard function (hf) is given

$$
h(x ; \alpha, \beta)=\alpha \beta^{-\alpha} x^{\alpha-1},
$$


which can be increase, decrease or constant depending on , $\alpha>1, \alpha<1$ or $\alpha=1$, respectively.

The curves of the pdf, cdf, sf and hf of Weibull distribution are shown in Fig. 1 for selected parameter values.

\section{Percentile Method (PM)}

The quantile function corresponding to Eqn. (1) is

$$
x_{p}=\beta[-\ln (1-p)]^{1 / \alpha} .
$$

Percentile estimations of the Weibull distribution are calculated based on the obtained equations from selected two percentile points in the quantile function. The percentile estimators of the shape and scale parameters for Weibull distribution are respectively given by

$$
\begin{aligned}
& \hat{\alpha}=\frac{\ln \left[-\ln \left(1-p_{1}\right)\right]-\ln \left[-\ln \left(1-p_{2}\right)\right]}{\ln \left(x_{p_{1}}\right)-\ln \left(x_{p_{2}}\right)}, \\
& \hat{\beta}=\frac{x_{p_{1}}}{\left(-\ln \left(1-p_{1}\right)\right)^{1 / \hat{\alpha}}},
\end{aligned}
$$

where $p_{1}$ and $p_{2}$ are selected two percentile points and $x_{p_{1}}$ and $x_{p_{2}}$ are observation values corresponding to $p_{1}$ and $p_{2}$, respectively. There are many approaches for selection of the $p_{1}$ and $p_{2}$. For example, we can obtain an estimate for scale parameter by using one of these approaches like setting $p_{1}=1-\exp (-1) \cong 0.632$ (63.2th percentile point) into Eqn. (7). In this approach, the percentile estimators for Weibull distribution are given

$$
\begin{aligned}
& \hat{\beta}=x_{1-\exp (-1),} \\
& \hat{\alpha}=\left(\frac{\ln \left[-\ln \left(1-p_{2}\right)\right]}{\ln \left(x_{p_{2}}\right)-\ln (\widehat{\beta})}\right),
\end{aligned}
$$

where $0<x_{p_{2}}<x_{0.632}$ [7]. There are some suggestions for the optimum value of $p_{2}$. Wang and Keats [5] showed by simulation that 0.15 would be the correct choice for $p_{2}$. Seki and Yokoyama [8] suggested $p_{2}=0.31$.

The percentile based estimators for $\alpha$ and $\beta$ of Weibull distribution according to approach 2 can be obtained by minimizing

$$
\sum_{i=1}^{n}\left\{\ln x_{(i)}-\ln \beta-\frac{1}{\alpha} \ln \left(-\ln \left(1-\widehat{F}_{(i)}\right)\right\}^{2}\right.
$$


with respect to $\alpha$ and $\beta$. The percentile estimators for $\alpha$ and $\beta$ of the Weibull distribution are given by

$$
\begin{aligned}
& \hat{\alpha}=\frac{A^{2}-n B}{A C-n D}, \\
& \hat{\beta}=\exp \left(\frac{1}{n}\left(C-\frac{A}{\hat{\alpha}}\right)\right),
\end{aligned}
$$

where $A=\sum_{i=1}^{n} \ln \left(-\ln \left(1-\hat{F}_{(i)}\right)\right), B=\sum_{i=1}^{n}\left\{\ln \left(-\ln \left(1-\hat{F}_{(i)}\right)\right)\right\}^{2}, C=\sum_{i=1}^{n} \ln x_{i}$ and $D=$ $\sum_{i=1}^{n} \ln x_{i} \ln \left(-\ln \left(1-\hat{F}_{(i)}\right)\right)$.

\section{Simulation Study}

In this study, we designed a simulation according to different sample size and different parameter values with the following purposes:

- To determine the best empirical distribution function respect to $M S E$ for approach 1 in the percentile method.

- To choose the best selected second percentile point $p_{2}$ when setting $p_{1}=1-\exp (-1) \cong$ 0.632 for approach 1 .

- To determine the best percentile pair among from several selected percentile pairs for approach 1 in the percentile estimation.

- To determine the best empirical distribution function for the best successful estimation according to MTE in the percentile estimation by approach 2 .

Table 1: The selected empirical distribution functions

\begin{tabular}{ll}
\hline Kaplan-Meir & $\hat{F}_{1}\left(x_{(i)}\right)=\frac{i}{n}$ \\
\hline Herd-Johnson (Mean rank) & $\hat{F}_{2}\left(x_{(i)}\right)=\frac{i}{n+1}$ \\
\hline Median (Symmetrical) & $\hat{F}_{3}\left(x_{(i)}\right)=\frac{i-0.5}{n}$ \\
\hline Median rank & $\hat{F}_{4}\left(x_{(i)}\right)=\frac{i-0.3}{n+0.4}$ \\
\hline Approximate Normal & $\hat{F}_{5}\left(x_{(i)}\right)=\frac{i-\frac{3}{8}}{n+\frac{1}{4}}$ \\
\hline
\end{tabular}

Firstly, we examined fitness between empirical distribution functions and actual cumulative distribution function values of the Weibull distribution for approach 1 in the percentile estimation method. The selected EDFs are given in Table 1. 
We used mean squared error $(M S E)$ in the comparison of the fitness between empirical distribution functions and actual cumulative distribution function values of Weibull distribution for the different parameter values and sample sizes.

$$
M S E_{m}=\frac{1}{100000} \sum_{r=1}^{100000} \sum_{i=1}^{n} \frac{\left[F\left(x_{(i)} ; \alpha, \beta\right)-\hat{F}_{m}\left(x_{(i)}\right)\right]^{2}}{n}
$$

In the simulation study, 100000 random samples of sizes $10,30,50$ and 100 were generated from Weibull distributions having $\alpha=0.5,1,1.5,2,3.4,5$ and $\beta=1,10$. The comparisons of the empirical distribution functions respect to MSE are given in Table 2.

In accordance with Table 2, the empirical distribution function that best matches the actual cumulative distribution function values of the Weibull distribution is Hard-Johnson's empirical distribution function for all the cases. Hard-Johnson's function will be used as empirical distribution function in the determination of the best percentile pairs for the percentile estimations according to approach 1 .

At this stage of the study, we will try to determine the best percentile point for the $p_{1}=$ $1-\exp (-1) \cong 0.632$ in the approach 1 . For this, the $\operatorname{MSE}(\hat{\alpha})$ values for all candidate points were determined with 0.01 increases from 0 to 0.63 .

$$
\operatorname{MSE}(\hat{\alpha})=\frac{1}{10000} \sum_{r=1}^{10000}\left(\alpha-\hat{\alpha}_{r}\right)^{2}
$$

In the simulated study for the different parameter values and sample sizes, the percentile point with the smallest $\operatorname{MSE}(\hat{\alpha})$ was determined, and the results are given in Table 3.

Table 2: The comparison of the EDFs according to MSE for different sample sizes and parameter values

\begin{tabular}{cccccccccccc}
\hline & & \multicolumn{1}{c}{$\boldsymbol{\beta}=\mathbf{1}$} & \multicolumn{6}{c}{$\boldsymbol{\beta}=\mathbf{1 0}$} \\
\cline { 2 - 11 } $\boldsymbol{\alpha}$ & $\boldsymbol{n}$ & $\widehat{\boldsymbol{F}}_{\mathbf{1}}$ & $\widehat{\boldsymbol{F}}_{\mathbf{2}}$ & $\widehat{\boldsymbol{F}}_{\mathbf{3}}$ & $\widehat{\boldsymbol{F}}_{\mathbf{4}}$ & $\widehat{\boldsymbol{F}}_{\mathbf{5}}$ & $\widehat{\boldsymbol{F}}_{\mathbf{1}}$ & $\widehat{\boldsymbol{F}}_{\mathbf{2}}$ & $\widehat{\boldsymbol{F}}_{\mathbf{3}}$ & $\widehat{\boldsymbol{F}}_{\mathbf{4}}$ & $\widehat{\boldsymbol{F}}_{\mathbf{5}}$ \\
\hline \multirow{4}{*}{$\mathbf{0 . 5}$} & 10 & 0.01825 & $\mathbf{0 . 0 1 5 0 3}$ & 0.01567 & 0.01523 & 0.01537 & 0.01830 & $\mathbf{0 . 0 1 5 2 2}$ & 0.01588 & 0.01544 & 0.01557 \\
& 30 & 0.00583 & $\mathbf{0 . 0 0 5 4 8}$ & 0.00556 & 0.00551 & 0.00552 & 0.00573 & $\mathbf{0 . 0 0 5 3 7}$ & 0.00546 & 0.00540 & 0.00542 \\
& 50 & 0.00342 & $\mathbf{0 . 0 0 3 2 8}$ & 0.00332 & 0.00330 & 0.00330 & 0.00342 & $\mathbf{0 . 0 0 3 2 8}$ & 0.00331 & 0.00329 & 0.00330 \\
& 100 & 0.00167 & $\mathbf{0 . 0 0 1 6 3}$ & 0.00164 & 0.00164 & 0.00164 & 0.00168 & $\mathbf{0 . 0 0 1 6 5}$ & 0.00166 & 0.00166 & 0.00166 \\
\hline & 10 & 0.01851 & $\mathbf{0 . 0 1 5 3 4}$ & 0.01604 & 0.01558 & 0.01572 & 0.01842 & $\mathbf{0 . 0 1 5 3 0}$ & 0.01595 & 0.01551 & 0.01564 \\
$\mathbf{1}$ & 30 & 0.00578 & $\mathbf{0 . 0 0 5 3 9}$ & 0.00549 & 0.00543 & 0.00545 & 0.00574 & $\mathbf{0 . 0 0 5 3 7}$ & 0.00545 & 0.00540 & 0.00542 \\
& 50 & 0.00340 & $\mathbf{0 . 0 0 3 2 6}$ & 0.00329 & 0.00327 & 0.00328 & 0.00342 & $\mathbf{0 . 0 0 3 2 9}$ & 0.00332 & 0.00330 & 0.00330 \\
& 100 & 0.00169 & $\mathbf{0 . 0 0 1 6 6}$ & 0.00167 & 0.00167 & 0.00167 & 0.00168 & $\mathbf{0 . 0 0 1 6 5}$ & 0.00165 & 0.00165 & 0.00165 \\
\hline & 10 & 0.01798 & $\mathbf{0 . 0 1 4 7 9}$ & 0.01545 & 0.01500 & 0.01514 & 0.01837 & $\mathbf{0 . 0 1 5 2 2}$ & 0.01595 & 0.01547 & 0.01562 \\
& 30 & 0.00574 & $\mathbf{0 . 0 0 5 3 4}$ & 0.00544 & 0.00538 & 0.00540 & 0.00571 & $\mathbf{0 . 0 0 5 3 2}$ & 0.00542 & 0.00536 & 0.00538 \\
$\mathbf{1} \mathbf{1 . 5}$ & 50 & 0.00339 & $\mathbf{0 . 0 0 3 2 5}$ & 0.00328 & 0.00326 & 0.00326 & 0.00346 & $\mathbf{0 . 0 0 3 3 2}$ & 0.00335 & 0.00333 & 0.00334 \\
& 100 & 0.00166 & $\mathbf{0 . 0 0 1 6 2}$ & 0.00163 & 0.00163 & 0.00163 & 0.00168 & $\mathbf{0 . 0 0 1 6 5}$ & 0.00166 & 0.00165 & 0.00166 \\
\hline
\end{tabular}




\begin{tabular}{cccccccccccc}
\hline & 10 & 0.01822 & $\mathbf{0 . 0 1 5 1 2}$ & 0.01577 & 0.01533 & 0.01546 & 0.01829 & $\mathbf{0 . 0 1 5 0 8}$ & 0.01573 & 0.01529 & 0.01542 \\
$\mathbf{2}$ & 30 & 0.00577 & $\mathbf{0 . 0 0 5 4 3}$ & 0.00551 & 0.00546 & 0.00547 & 0.00564 & $\mathbf{0 . 0 0 5 3 0}$ & 0.00539 & 0.00533 & 0.00535 \\
& 50 & 0.00337 & $\mathbf{0 . 0 0 3 2 4}$ & 0.00327 & 0.00325 & 0.00326 & 0.00342 & $\mathbf{0 . 0 0 3 3 1}$ & 0.00334 & 0.00332 & 0.00333 \\
& 100 & 0.00167 & $\mathbf{0 . 0 0 1 6 4}$ & 0.00165 & 0.00165 & 0.00165 & 0.00169 & $\mathbf{0 . 0 0 1 6 6}$ & 0.00166 & 0.00166 & 0.00166 \\
\hline \multirow{4}{*}{$\mathbf{3 . 4}$} & 10 & 0.01833 & $\mathbf{0 . 0 1 5 3 0}$ & 0.01598 & 0.01553 & 0.01567 & 0.01829 & $\mathbf{0 . 0 1 5 0 2}$ & 0.01573 & 0.01526 & 0.01541 \\
& 30 & 0.00578 & $\mathbf{0 . 0 0 5 4 0}$ & 0.00549 & 0.00543 & 0.00545 & 0.00578 & $\mathbf{0 . 0 0 5 4 1}$ & 0.00549 & 0.00543 & 0.00545 \\
& 50 & 0.00344 & $\mathbf{0 . 0 0 3 3 1}$ & 0.00335 & 0.00333 & 0.00333 & 0.00340 & $\mathbf{0 . 0 0 3 2 7}$ & 0.00330 & 0.00328 & 0.00329 \\
& 100 & 0.00167 & $\mathbf{0 . 0 0 1 6 4}$ & 0.00164 & 0.00164 & 0.00164 & 0.00167 & $\mathbf{0 . 0 0 1 6 3}$ & 0.00164 & 0.00163 & 0.00163 \\
\hline \multirow{4}{*}{$\mathbf{5}$} & 10 & 0.01833 & $\mathbf{0 . 0 1 5 2 0}$ & 0.01589 & 0.01543 & 0.01557 & 0.01859 & $\mathbf{0 . 0 1 5 2 9}$ & 0.01599 & 0.01553 & 0.01567 \\
& 30 & 0.00570 & $\mathbf{0 . 0 0 5 3 6}$ & 0.00545 & 0.00539 & 0.00541 & 0.00573 & $\mathbf{0 . 0 0 5 3 5}$ & 0.00544 & 0.00538 & 0.00540 \\
& 50 & 0.00346 & $\mathbf{0 . 0 0 3 3 2}$ & 0.00335 & 0.00333 & 0.00334 & 0.00340 & $\mathbf{0 . 0 0 3 2 7}$ & 0.00330 & 0.00328 & 0.00329 \\
& 100 & 0.00168 & $\mathbf{0 . 0 0 1 6 5}$ & 0.00166 & 0.00165 & 0.00165 & 0.00170 & $\mathbf{0 . 0 0 1 6 7}$ & 0.00168 & 0.00167 & 0.00167 \\
\hline
\end{tabular}

Table 3: The percentile points values which have the smallest $\operatorname{MSE}(\widehat{\alpha})$ for different sample sizes and parameter values

\begin{tabular}{cccccccccccccc}
\hline \multirow{2}{*}{$\begin{array}{c}\text { Sample } \\
\text { Size }\end{array}$} & \multicolumn{1}{c}{$\boldsymbol{\beta}=\mathbf{1}$} \\
\cline { 2 - 6 } $\boldsymbol{n}$ & $\mathbf{0 . 5}$ & $\mathbf{1}$ & $\mathbf{1 . 5}$ & $\mathbf{2}$ & $\mathbf{3 . 4}$ & $\mathbf{5}$ & $\mathbf{0 . 5}$ & $\mathbf{1}$ & $\mathbf{1 . 5}$ & $\mathbf{2}$ & $\mathbf{3 . 4}$ & $\mathbf{5}$ \\
\hline $\mathbf{1 0}$ & 0.13 & 0.13 & 0.13 & 0.13 & 0.13 & 0.13 & 0.13 & 0.13 & 0.13 & 0.13 & 0.13 & 0.13 \\
$\mathbf{3 0}$ & 0.11 & 0.14 & 0.14 & 0.14 & 0.14 & 0.14 & 0.14 & 0.14 & 0.14 & 0.14 & 0.14 & 0.14 \\
$\mathbf{5 0}$ & 0.12 & 0.12 & 0.12 & 0.14 & 0.10 & 0.12 & 0.12 & 0.10 & 0.12 & 0.14 & 0.10 & 0.14 \\
$\mathbf{1 0 0}$ & 0.11 & 0.12 & 0.11 & 0.12 & 0.12 & 0.13 & 0.14 & 0.13 & 0.12 & 0.13 & 0.13 & 0.12 \\
\hline
\end{tabular}

According to Table 3, the most suitable percentile points is found ranges 0.10 to 0.14 for the $p_{1} \cong 0.632$ in the approach 1 . The best percentile point was 0.13 with respect to $M S E(\hat{\alpha})$ in the shape parameter estimation of the Weibull distribution by this approach when the small sample size is $n=10$.

Here, the best percentile pair will be determined from the selected percentile pairs in the percentile estimation by approach 1 of the Weibull distribution parameters. The comparison is based on MTE criterion defined by

$$
M T E=\frac{1}{10000} \sum_{r=1}^{10000}\left(\left(\frac{\widehat{\alpha}_{r}-\alpha}{\alpha}\right)^{2}+\left(\frac{\widehat{\beta}_{r}-\beta}{\beta}\right)^{2}\right)
$$

In this study, the selected percentile pairs are denoted in Table 4.

Table 4: The selected pairs of percentage point

\begin{tabular}{cccc}
\hline$A: P\left(\frac{1}{n+1}, \frac{n}{n+1}\right)$ & $B: P(0.25,0.75)$ & $C: P\left(0.25, \frac{n}{n+1}\right)$ & $D: P\left(\frac{1}{n+1}, 0.75\right)$ \\
\hline$E: P\left(0.5, \frac{n}{n+1}\right)$ & $F: P\left(\frac{1}{n+1}, 0.5\right)$ & $G: P(0.632,0.30)$ & $H: P(0.05,0.95)$ \\
\hline$I: P(0.10,0.90)$ & $J: P(0.17,0.97)$ & $K: P(0.24,0.93)$ & $L: P(0.632,0.12)$ \\
\hline
\end{tabular}


In the simulation study, the obtained $M T E$ values for different parameter values and sample sizes are given in Table 5.

According to Table 5, in case of the scale parameter is equal $1, J$ and $K$ percentile pairs point are more successful than other percentile pairs for $\geq 1$. The $B$ percentile pair is more successful than other pairs for $\alpha<1$ and $\beta=1$. In case of the scale parameter is set as $\beta=10$, $B$ percentile pair is more successful than other percentile pairs for $\alpha \leq 1$. The $K$ percentile pair is more successful than other pairs for $\alpha=2$ and $\beta=10$. In addition, $\mathrm{J}$ percentile pair showed more performance than other pairs for $\alpha=5$ and $\beta=10$.

Finally, we performed a simulation study to investigate the performance of empirical distribution functions in the percentile estimates for parameters of the Weibull distribution by approach 2. The comparison results of the empirical distribution functions according to $M T E$ for different sample sizes and parameter values in the approach 2 are given in Table 6.

Table 5: The comparison results of the selected percentage points according to $M T E$

\begin{tabular}{|c|c|c|c|c|c|c|c|c|c|c|c|c|c|c|}
\hline$\beta$ & $\alpha$ & $n$ & $\mathbf{A}$ & B & C & D & $\mathbf{E}$ & $\mathbf{F}$ & G & I & I & $\mathbf{J}$ & 1 & L \\
\hline & \multirow{4}{*}{0} & 1 & 725 & 201 & 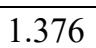 & & 405 & 7 & 617 & 5 & 5 & .538 & 6 & 6 \\
\hline & & 30 & & 65 & 18 & 8 & 320 & 478 & 0.394 & 57 & 48 & 4 & 8 & 0.408 \\
\hline & & 50 & $=0$ & 150 & 262 & 178 & 0.182 & 530 & 176 & 0.260 & .186 & 226 & 165 & 177 \\
\hline & & 100 & & & & & 0.091 & & & & & & & 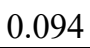 \\
\hline & & 10 & & 462 & J & & 0 & & 428 & & 41 & & 15 & 552 \\
\hline & & 30 & & 109 & 04 & & 0.106 & & & & 0.103 & & & 0.160 \\
\hline & & 50 & 1 & 064 & 068 & 0 & 0.066 & 55 & 75 & 0.077 & .061 & & & 0.075 \\
\hline & & 100 & 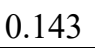 & 0.031 & . & 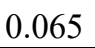 & 6 & 7 & 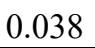 & 12 & 0.030 & & 27 & 0.046 \\
\hline & \multirow{4}{*}{1.5} & 10 & 4 & .523 & 1 & 0.33 & 0 & & & 4 & 0.264 & 50 & 1 & 0 \\
\hline & & 30 & & 1 & 77 & 0.139 & 0.098 & 0.237 & 26 & 0.096 & 0.084 & 81 & 73 & 0.168 \\
\hline & & 50 & 117 & 065 & 050 & 0.101 & 0.062 & 0.146 & .075 & 0.059 & 0.051 & 46 & 0.045 & 0.076 \\
\hline & & 100 & Jy & 030 & 29 & 0.076 & 0.0 & 7 & 1 & 3 & 0.025 & & 3 & 0.049 \\
\hline & \multirow{4}{*}{2} & 10 & 279 & 7 & 20 & & 0 . & 0.6 & 13 & & 0.279 & & & 0.752 \\
\hline & & 30 & & & & & & & & & & & & \\
\hline & & 50 & 0.11 & 076 & 050 & 0.1 & 0.071 & 0. & 0 & 0.0 & 0.055 & 17 & 0.049 & 0.089 \\
\hline & & 100 & 3 & .035 & 029 & 0. & 0.039 & 0.127 & 43 & 0.034 & 0.028 & & 0.024 & 0.059 \\
\hline & \multirow{4}{*}{3.} & 10 & 5 & 2 & 164 & & 1.262 & & & & 0.405 & & 04 & 1.220 \\
\hline & & 30 & 191 & 207 & 112 & 0.264 & 0.172 & 0.408 & 0.230 & 0.141 & 0.131 & 07 & 0.113 & 0.324 \\
\hline & & 50 & & & & & & & & & & & & 0.140 \\
\hline & & 100 & & & & & & & & & 0.041 & & & 0.094 \\
\hline & \multirow{4}{*}{5} & 10 & & & & & & & & & & & & 1.780 \\
\hline & & 30 & 0 & 0.300 & 0.150 & 0. & 0.249 & 0.5 & 33 & 0.200 & 0.188 & & 0.161 & 0.472 \\
\hline & & 50 & ح & 0.174 & 0.099 & 0.2 & 0.159 & 0.3 & 1 & 0.1 & 0.114 & 92 & 0 . & 0.203 \\
\hline & & 100 & 3 & 080 & $0=r$ & & 0.0 & & & & 0.058 & & 0.0 & 0.137 \\
\hline \multirow{4}{*}{10} & \multirow{4}{*}{0.5} & 10 & & & & & & & & & & & & 14.45 \\
\hline & & 30 & & & & & 2.950 & & & & 3.298 & 95 & & 3.661 \\
\hline & & 50 & & & & & & & & & 1.679 & 2.083 & 1.498 & 1.525 \\
\hline & & 100 & 0.311 & 0.602 & 1.454 & 0.796 & 0.804 & 2.826 & 0.179 & 1.204 & 0.781 & 1.018 & 0.707 & 0.775 \\
\hline
\end{tabular}




\begin{tabular}{|c|c|c|c|c|c|c|c|c|c|c|c|c|c|}
\hline \multirow{4}{*}{1} & 10 & & & & 19 & 33 & 5.521 & 2.508 & 2.000 & 2.000 & 2. & O & \\
\hline & 30 & & 549 & 0.764 & 621 & 617 & 753 & 765 & 878 & 692 & .906 & .641 & \\
\hline & 50 & 321 & 315 & 0.491 & 0.401 & 0.368 & 0.847 & 393 & .540 & 0.390 & 0.442 & .343 & \\
\hline & 100 & 115 & 156 & 0.305 & 243 & 0.194 & .537 & 202 & .288 & 193 & .233 & .173 & דרו1. \\
\hline \multirow{4}{*}{1.5} & 10 & 1.091 & 139 & 0.911 & 34 & 1.245 & 1.679 & 630 & 1.091 & 091 & 949 & 911 & \\
\hline & 30 & 0.717 & 0.305 & 0.351 & 0.354 & 0.315 & 0.785 & 0.426 & 0.422 & 339 & .404 & 307 & $3 / 1$ \\
\hline & 50 & 0.629 & 0.174 & 0.227 & 0.240 & 0.192 & 0.423 & 0.234 & 0.263 & 0.195 & 0.208 & 0.171 & 208 \\
\hline & 100 & 0.538 & 0.086 & 0.140 & 0.156 & 0.102 & 0.279 & 0.117 & 0.140 & 0.097 & 0.111 & 0.087 & 0.105 \\
\hline \multirow{4}{*}{2} & 10 & 0.738 & 1.021 & 0.648 & 0.731 & 1.138 & 1.297 & 1.567 & 0.738 & 0.738 & 0.649 & 0.648 & 0.898 \\
\hline & 30 & 0.467 & 0.244 & 0.230 & 0.286 & 0.233 & 0.549 & 0.353 & 0.283 & 0.234 & 0.257 & 0.208 & 0.287 \\
\hline & 50 & 0.407 & 0.137 & 0.148 & 0.202 & 0.145 & 0.319 & 0.198 & 0.176 & 0.136 & 0.137 & 0.120 & 0.164 \\
\hline & 100 & 0.347 & 0.067 & 0.090 & 0.138 & 0.077 & 0.217 & 0.097 & 0.094 & 0.067 & 0.073 & 0.060 & 0.081 \\
\hline \multirow{4}{*}{3.4} & 10 & 562 & 4 & 0.573 & 0.748 & 1.443 & 1.3 & 2.099 & 0.562 & 62 & 26 & .573 & .961 \\
\hline & 30 & 0.305 & 0.257 & 0.165 & 0.301 & 0.217 & 0.487 & 0.395 & 0.207 & 0.183 & 70 & 60 & 83 \\
\hline & 50 & 0.255 & 0.141 & 0.105 & 0.224 & 0.138 & 0.315 & 0.225 & 0.128 & 0.109 & .096 & 0.098 & 167 \\
\hline & 100 & 0.209 & 0.068 & 0.060 & 0.164 & 0.074 & 0.226 & 0.107 & 0.067 & 0.054 & 50 & 0.048 & 0.080 \\
\hline \multirow{4}{*}{5} & 10 & 645 & 1.740 & 0.712 & 0.973 & 1.999 & 1.810 & 2.945 & 0.645 & 645 & 28 & .712 & 71 \\
\hline & 30 & 0.318 & 0.338 & 0.187 & 0.395 & 0.274 & 0.610 & 0.529 & 0.233 & 0.215 & 0.184 & 0.186 & 0.364 \\
\hline & 50 & 0.256 & 0.183 & 0.117 & 0.299 & 0.176 & 0.409 & 0.302 & 0.143 & 0.128 & 0.107 & 0.117 & 0.217 \\
\hline & 100 & 204 & 0.088 & 0.065 & 0.223 & 0.093 & 0.142 & 0.074 & 0.063 & 0.063 & 0.055 & 0.057 & 0.103 \\
\hline
\end{tabular}

Table 6: The comparison results of the empirical distribution functions according to $M T E$ for different sample sizes and parameter values in the approach 2

\begin{tabular}{|c|c|c|c|c|c|c|c|c|c|c|c|}
\hline \multirow[t]{2}{*}{$\alpha$} & \multirow[t]{2}{*}{$n$} & \multicolumn{5}{|c|}{$\beta=1$} & \multicolumn{5}{|c|}{$\beta=10$} \\
\hline & & $\widehat{F}_{1}$ & $\widehat{F}_{2}$ & $\widehat{F}_{3}$ & $\widehat{F}_{4}$ & $\widehat{F}_{5}$ & $\widehat{F}_{1}$ & $\widehat{F}_{2}$ & $\widehat{F}_{3}$ & $\widehat{F}_{4}$ & $\widehat{F}_{5}$ \\
\hline \multirow{4}{*}{0.} & 10 & 0.6209 & 1.2054 & 0.8843 & 0.8976 & 0.9490 & 5.6093 & 11.584 & 8.2614 & 7.0162 & 8.9809 \\
\hline & 30 & 0.1872 & 0.2579 & 0.2137 & 0.2210 & 0.2236 & 1.6950 & 2.4080 & 1.9811 & 1.8365 & 2.0847 \\
\hline & 50 & 0.1157 & 0.1439 & 0.1238 & 0.1278 & 0.1284 & 1.0498 & 1.3319 & 1.1438 & 1.0858 & 1.1906 \\
\hline & 100 & 0.0563 & 0.0647 & 0.0579 & 0.0595 & 0.0594 & 0.5066 & 0.5902 & 0.5293 & 0.5127 & 0.5445 \\
\hline & 10 & 0.2533 & 2632 & 2600 & 0.2384 & 0.2500 & 1.3328 & 1.6937 & 1.4358 & 1.1833 & 1.4813 \\
\hline & 30 & 0.0791 & 0.0840 & 0755 & 0.0751 & 0.0757 & 0.4369 & 0.4981 & 0.4433 & 0.4030 & 0.4545 \\
\hline & 50 & 0.0485 & 0.0511 & 0.0457 & 0.0461 & 0.0461 & 0.2704 & 0.2972 & 0.2696 & 0.2523 & 0.2753 \\
\hline & 100 & 0.0247 & 0.0258 & 0.0232 & 0.0236 & 0.0235 & 0.1341 & 0.1440 & 0.1332 & 0.1277 & 0.1354 \\
\hline \multirow{4}{*}{1.5} & 1 & 2 & 86 & 479 & 47 & 0.2254 & 22 & 78 & 0.7 & & 0.7262 \\
\hline & 30 & 0.0770 & 0.0762 & 0.0696 & 0.0683 & 0.0684 & 0.2385 & 0.2494 & 0.2277 & 0.2120 & 0.2300 \\
\hline & 50 & 0.0468 & 0.0473 & 0.0421 & 0.0422 & 0.0420 & 0.1458 & 0.1518 & 0.1391 & 0.1330 & 0.1407 \\
\hline & 100 & 0.0242 & 0.0247 & 0.0218 & 0.0222 & 0.0220 & 0.0728 & 0.0758 & 0.0700 & 0.0683 & 0.0709 \\
\hline & 10 & 0.2998 & 0.2418 & 0.2884 & 0.2444 & 0.2567 & 0.5974 & 0.5413 & 0.5556 & 0.4810 & 0.5305 \\
\hline & 30 & 0.0889 & 0.0864 & 0.0791 & 0.0773 & 0.0772 & 0.1810 & 0.1815 & 0.1671 & 0.1610 & 0.1668 \\
\hline & 50 & 0.0540 & 0.0540 & 0.0477 & 0.0479 & 0.0474 & 0.1099 & 0.1117 & 0.1018 & 0.1004 & 0.1023 \\
\hline & 100 & 0.0281 & 0.0285 & 0.0249 & 0.0254 & 0.0251 & 0.0555 & 0.0569 & 0.0519 & 0.0519 & 0.0524 \\
\hline \multirow{3}{*}{3.4} & 10 & 4657 & 3656 & 00 & 0 & 0.3950 & 33 & 45 & 0.5 & 8 & 79 \\
\hline & 30 & 0.1374 & 1326 & 0.1212 & 0.1182 & 77 & 0.1700 & 0.1646 & 0.1 & 0. & 0.1 \\
\hline & 50 & 0.0834 & 0831 & 0.0730 & 0.0732 & 0.0724 & 0.1030 & 0.1026 & 0.0916 & 0.0949 & 0.0911 \\
\hline
\end{tabular}




\begin{tabular}{cccccccccccc}
\hline & 100 & 0.0436 & 0.0442 & $\mathbf{0 . 0 3 8 3}$ & 0.0391 & 0.0385 & 0.0531 & 0.0539 & $\mathbf{0 . 0 4 7 6}$ & 0.0497 & 0.0479 \\
\hline \multirow{4}{*}{5} & 10 & 0.6727 & $\mathbf{0 . 5 2 6 4}$ & 0.6515 & 0.5433 & 0.5705 & 0.7258 & $\mathbf{0 . 5 7 1 5}$ & 0.6938 & 0.6165 & 0.6133 \\
& 30 & 0.1984 & 0.1913 & 0.1749 & 0.1705 & $\mathbf{0 . 1 6 9 7}$ & 0.2137 & 0.2060 & 0.1888 & 0.1932 & $\mathbf{0 . 1 8 3 8}$ \\
& 50 & 0.1205 & 0.1200 & 0.1053 & 0.1056 & $\mathbf{0 . 1 0 4 3}$ & 0.1296 & 0.1289 & 0.1138 & 0.1189 & $\mathbf{0 . 1 1 2 9}$ \\
& 100 & 0.0631 & 0.0639 & $\mathbf{0 . 0 5 5 2}$ & 0.0565 & 0.0556 & 0.0675 & 0.0683 & $\mathbf{0 . 0 5 9 5}$ & 0.0627 & 0.0599 \\
\hline
\end{tabular}

In the large sample sizes $(n=50$ and 100), the difference between MTE values of the empirical distribution functions was not significant according to Table 6. In case of the shape parameter is set as $\alpha=0.5$, Kaplan-Meir's empirical distribution function has lower MTE value than other empirical distribution functions for $\beta=1$ and 10 .

\section{Conclusion}

In this paper, we aimed at improving the effectiveness of the percentile method for two parameter Weibull distribution. Based on simulation study, the empirical distribution function that best fits the actual Weibull cumulative distribution function is the empirical distribution function of Hard Johnson according to $M S E$. In conclusion, the most suitable percentage points ranged from 0.10 to 0.14 when $p_{1}$ value was nearly set as 0.632 in the percentile method by approach 1 . In general, $(0.17,0.97)$ and $(0.24,0.93)$ percentile pairs were the best successful percentile pairs for $\alpha \geq 1$. In case of the $\alpha<1,(0.25,0.75)$ percentile pair was more successful than other percentile pairs. Also, median (symmetrical) empirical distribution function has been more successful in case of large sample size $(n=100)$ for percentile method by approach 2 .

\section{References}

[1] Castillo, E., Hadi, A.S., A method for estimating parameters and quantiles of distributions of continuous random variables, Computational Statistics \& Data Analysis, 20(4), 421-439, 1995.

[2] Erisoğlu, U., Erisoğlu, M., Percentile estimators for two-component mixture distribution models, Iranian Journal of Science and Technology, Transactions A: Science, 43(2), 601-619, 2019.

[3] Dubey, S.D., Some percentile estimators for Weibull parameters, Technometrics, 9(1), 119-129, 1967.

[4] Seki, T., Yokoyama, S., Simple and robust estimation of the Weibull parameters, Microelectronics Reliability, 33(1), 45-52, 1993.

[5] Wang, F.K., Keats, J.B., Improved percentile estimation for the two-parameter Weibull distribution, Microelectronics Reliability, 35(6), 883-892, 1995.

[6] Marks, N.B., Estimation of Weibull parameters from common percentiles, Journal of Applied Statistics, 32(1), 17-24, 2005. 
[7] Teimouri, M., Hoseini, S.M., Nadarajah, S., Comparison of estimation methods for the Weibull distribution, Statistics, 47(1), 93-109, 2013.

[8] Seki, T., Yokoyama, S., Robust parameter-estimation using the bootstrap method for the 2-parameter Weibull distribution, IEEE Transactions on Reliability, 45(1), 34-41, 1996. 